\title{
Rules of Three Untrained Workers' Assignment Optimization in Reset Limited-Cycled Model with Multiple Periods
}

\author{
Peiya Song \\ Graduate School of System Design, Tokyo Metropolitan University, Tokyo, Japan \\ Xianda Kong*, Hisashi Yamamoto \\ Faculty of System Design, Tokyo Metropolitan University, Tokyo, Japan \\ Jing Sun \\ Faculty of Engineering, Nagoya Institute of Technology, Nagoya, Japan \\ Masayuki Matsui \\ Faculty of Engineering, Kanagawa University, Yokohama, Japan
}

(Received: January 29, 2015 / Revised: August 25, 2015; November 27, 2015 / Accepted: December 18, 2015)

\begin{abstract}
In labor-intensive enterprise, such as garment factory, assembly line is widely used as a manufacturing process for reducing costs and production time. However, for the sake of the various working capacity of worker, idle or delay may happen and influence the rear processes. If these unforeseeable delay happened continuously, it may influence the whole manufacturing process and a model, which is called limited-cycle model with multiple periods (LCMwMP), is assumed to evaluate the influence risk. In order to minimize the risk, the assignment of the workers is focused on. In this paper, we deal with an assembly line as LCMwMP model when two kinds of workers exist, whose efficiency is assumed to two different groups. We consider an optimization problem for finding an assignment of workers to the line that minimizes total expected risk, which is exchanged to expected cost by reset model of LCMwMP. First, reset model as a simple model of LCMwMP is introduced. Then, some hypotheses of the rules of the optimal worker assignment are proposed and some numerical experiments are researched assuming the processing time as Erlang distribution. Finally, the other rules on other certain conditions are discussed.
\end{abstract}

Keywords: Limited-Cycle Model, Multiple Periods, Optimal Assignment, Processing Efficiency

* Corresponding Author, E-mail: xianda.kong@gmail.com

\section{INTRODUCTION}

After Industrial Revolution in $18^{\text {th }}$ century, the development of the assembly line led to a proliferation of manufacturing inventions and contributed to the substantial fortunes. And till now, we can still benefit from assembly line, which is widely used among labor-intensive en- terprise, such as garment factory, for the sake of reducing processing costs and production time. However, even though the reliability of the machine on the assembly line in nowadays surpasses that of centuries ago, the each component of assembly line is still facing the problem of failure during the processing process. And not every worker on the assembly line can accomplish the task 
arranged in time. All these factors will lead to a production delay which can result in financial losses, if we consider the cost of production which fluctuates with the delay, to the manufacturing enterprise, solving the problem mentioned above is important for achieving the goal that processing task can be accomplished efficiently and competently. The efficiency can be lamed not only by the factor of the machines of the each component on the assembly line, but also by the factor of the workers, such as the difference of production speed between well-trained and untrained. The former is well known as the reliability of a system and the latter is considered as an assembly line balancing problem. And the latter is referred to as a problem of assignment under a limited-cycle model with multiple periods.

Since the first mathematical formalization of assembly line balancing by Salveson (1955) 60 years ago, assembly line balancing problem is popularly researched and developed (Nils et al., 2007). It assumes a homogeneous skill set to solve the problem of worker assignment in the above literature, that is to say, the skill of workers is not distinguished. Further, the properties of the optimal assignment are not developed. And Yamamoto et al. (2006) considered the solutions of this kind of problem.

In this problem, the characteristic in of the researches of Yamamoto et al. (2006) is the setting of constraint condition, (e.g., processing time with a target) which repeated in every multiple periods. If the constraint condition is broken through, an expected risk (e.g., penalty cost) will be occurred. Therefore the problem of minimizing the risk in such a situation is called limit-cycle model with multiple periods (LCMwMP). And the model of LCMwMP is used in this article.

In previous researches of Yamamoto et al. (2006, 2010), according whether the constraint condition (target processing time) reset or not, The LCMwMP is divided into reset model and non-reset model. In the field of reset model, a recursive formula for the total expected risk and an algorithm for optimal assignments based on the branch and bound method are proposed by Yamamoto et al. (2007). Recently, Yamamoto et al. (2011) and Kong et al. (2010) proposed properties of optimal worker assignment with two kinds of workers in which one special worker exists. Then, Kong et al. (2011a, 2011b) proposed properties of optimal worker assignment with two special workers.

After that Song et al. (2014) researched the properties of optimal assignment with two kinds of workers in which three special workers exist by the numerical experiment with exponential distribution.

In this paper, we will continuously discuss the properties by numerical experiment with Erlang distribution, and it indicates the similar properties. Therefore it inspires us that the rules of optimal arrangement problem are invariant in spite of various distributions. So by means of the numerical experiment, we will try to demonstrate the properties by theoretical proof in the future researches. In this paper, at first, reset model as a simple model of LCMwMP is introduced. Then, some hypotheses of the rules of the optimal worker assignment are proposed and some numerical experiments are conducted for demonstrating the hypotheses by assuming the processing time as Erlang distribution.

\section{MODEL EXPLANATION}

In this section, we consider a 'Reset model' which is a simple model of the LCMwMP. Then, we define the optimal assignment problem in reset model.

\subsection{Reset Model of Limited-Cycle Model with Multiple Periods}

The model is considered based on the following assumptions by Kong et al. (2010):

(1) In an assembly line system, $n$ is the number of process (it may be considered that $n$ is the number of processing station).

(2) The production is processed in a rotation of process 1 , process $2, \cdots$, and process $n$. One production will be processed by $n$ processes.

(3) All of the partly-finished productions will be moved to next process and established by time $Z$. Specifically $Z$ is the cycle time of all processes.

(4) There are two types of workers, $A$ and $B$. $A$ represents untrained worker whose processing rate is lower than others, $B$ represents regular worker. Note that assigning only one worker to each process.

(5) To sum up, $Z$ is the cycle time of all of the stations. $Z$ is also a kind of limited processing time (or target processing time) of each station. $Z$ is called the target processing time in this research.

$T_{l}$ : The processing time of worker $l$ which $l \in\{A, B\}$ :

$P_{l}$ : The probability of worker $l$ becoming idle, which is $\operatorname{Pr}\left\{T_{l} \leqq Z\right\}$,

$Q_{l}$ : The probability of the worker $l$ becoming delayed, which is $\operatorname{Pr}\left\{T_{l}>Z\right\}$,

$T S_{l}$ : The expected idle time of the worker $l$, which is $E\left[\left(Z-T_{l}\right) I\left(T_{l} \leqq Z\right)\right]$,

$T L_{l}$ : The expected delay time of the worker $l$, which is $E\left[\left(T_{l}-Z\right) I\left(T_{l} \leqq Z\right)\right]$,

where $I(O)$ is an index function and given as follows:

$$
I(O)= \begin{cases}1 & (O \text { is true }) \\ 0 & (O \text { is not true }) .\end{cases}
$$

We suppose the following costs as shown in Figure 1

In this paper, we consider a fixed target processing time $Z$. Workers' employment costs and resource will occur whether the processing is done. A processing cost $C_{t}(\geq 0)$ per unit time will proportionally occur to target processing time $Z$. Otherwise, if the processing time is longer than $Z$, overtime work or additional resources will be requested in order to meet the target time $Z$. So 


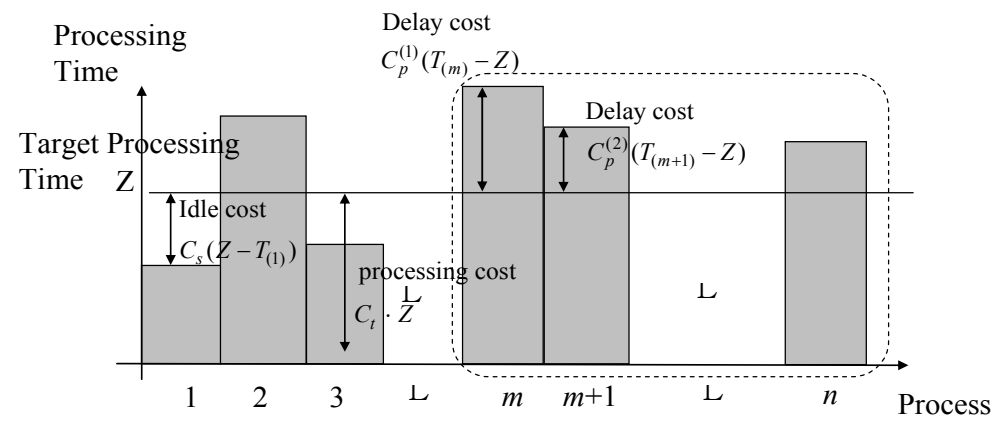

Figure 1. Description of idle and delay cost in reset model of LCMwMP.

the delay cost per unit time, $C_{P}^{(k)}(\geq 0)$, will occur (that is why we call the model a 'Reset Model'). Meanwhile, if the processing time is shorter than $Z$, in-process inventory can be considered before moving to the next process. So the idle cost per unit time, $C_{S}(\geq 0)$, will occur.

As a summary of above, we get:

(6) The processing cost per unit time, $C_{t}(\geq 0)$, for the target processing time limit occurs in each process.

(7) When $T_{l} \leq Z$, the idle cost per unit time, $C_{S}(\geq 0)$, occurs in each process. For example in Figure 1, in process 1 , the work is finished at time $T_{(1)}$, so during the time $T_{(l)}$ to $Z$, idle is happened and $C s$ is occurs per unit time.

(8) When $T_{l}>Z$, the delay cost per unit time, $C_{P}^{(k)}(\geq 0)$, occurs in the process if delay occurs in consecutive $k$ processes before its process, for $k=1,2, \mathrm{~L}, n$. In this paper, we suppose that the delayed process time of a process can be recovered by the overtime work or spare workers in this process, and $C_{P}^{(k)}$ is the cost for all of these. Because the cost rises due to the increase of the delay, in this paper, we suppose the $C_{P}^{(k)}$ is increasing in $k$, that is $0<C_{P}^{(1)} \mathrm{L}<C_{P}^{(n)}$.

We define $T_{(i)}$ is the actual processing time in process $i$, when $1 \leqq i \leqq n$. For example in Figure 1 , actual processing time $T_{(m)}$ is over the $Z$, so it can be known delay happens in process $m$, so during the time $Z$ to $T_{(m)}$, $C_{P}^{(1)}$ is also generated per unit time. And unfortunately, in the next process, the delay is continuously happened, so the penalty rises to $C_{P}^{(2)}$.

\subsection{Optimal Assignment Problem under Reset Model}

We consider that three untrained workers are allocated in this reset model. One of the most important problems is how to allocate workers to processes for minimizing the expected cost in $n$ processes. We call such a problem the optimal assignment problem. For describing the optimal assignment problem, we define the following notations (Kong et al., 2010):

For $1 \leq i<j \leq k \leq n$,

$\pi(i, j, k)$ : Three untrained workers are allocated in process $i, j$ and $k, n-3$ regular workers are allocated to processes out of process $i, j$ and process $k$.

$T C(n ; \pi(i, j, k))$ : The total costs of processes 1 to $n$ when workers are allocated by assignment $\pi(i, j, k)$.

The total cost $T C(n ; \pi(i, j, k))$ of $n$ processes when workers allocated according to assignment $\pi(i, j, k)$ is expressed as

$$
T C(n ; \pi(i, j, k))=n C_{t} Z+f(n ; \pi(i, j, k))
$$

where,

$f(n ; \pi(i, j, k))$ : The expected cost (the sum of the expected idle cost and the expected delay cost) caused in $n$ processes.

By using these notations, the optimal assignment problem with multiple periods becomes the problem of obtaining assignment in the following equation:

$$
T C\left(n ; \pi^{*}\right)=\min _{1 \leq i<j \leq k \leq n} T C(n ; \pi(i, j, k))
$$

In this paper, we call $\pi$ the optimal assignment.

However, it is easily known from (1) that if the target processing time $Z$ is constant, the target production cost, $n C_{t} Z$, is also constant, so we can simplify (2) to

$$
f\left(n ; \pi^{*}\right)=\min _{1 \leq i<j \leq k \leq n} f(n ; \pi(i, j, k))
$$

\section{HYPOTHESES}

\section{HYPOTHESIS 1:}

In the case of Song et al. (2014), when there is not a striking difference of processing rate between two kinds of workers, assignment $\pi(1,2,3)$ is the optimal worker assignment.

\section{HYPOTHESIS 2:}

When the difference becomes obvious, assignment 
$\pi(1,2, n)$ will be the optimal assignment. And when there is a striking difference, the optimal assignment will gradually transit to $\pi\left(1, \frac{1+n}{2}, n\right)$ (when $n$ is odd number) or $\pi\left(1, \frac{n}{2}, n\right)$ (when $n$ is even number).

According to the conclusion of the previous researches, it can be assumed that under the situation of Erlang distribution, the conclusion is more complicated but very similar to the result above.

The optimal assignments vary according to $C_{P}^{(i)}$, the quantities of consecutive delay cost, and the processing rate of these two kinds of worker, $\mu$, which is considered as manufacturing speed when the processing time of workers are followed exponential distribution. So in the following mathematical experiment, the relation between optimal assignment and the quantities of parameter $C_{P}^{(i)}$ and $\mu$ is examined.

Another aspect of the problem is to demonstrate the rule of optimal assignment on the basis of mathematic deduction. By shifting the allocation of each worker, the variation trend of expected cost can be easily summarized and it inspires the rule of optimal assignment of three untrained workers.

\section{MATHEMATICAL EXPERIMENTS}

The processing time of these two kinds of workers follows Erlang distribution. In other word, $Q_{l}$, the probability of the worker $l$ becoming delayed is

$$
Q_{l}=\sum_{k=0}^{m-1} \frac{\left(\mu_{l} Z\right)^{k}}{k !} e^{-\mu_{l} Z}
$$

where $l \in\{A, B\}$.

And $P_{l}$, the probability of the worker $l$ becoming idle, is $1-Q_{l}$.

Here is the description of the parameter of Erlang distribution.

(1) $\mu_{l}$ is the processing rate. And when $\mu_{l}$ is bigger, it means processing speed is higher and lower possibility of delay of processing.

(2) $m$ is the shape parameter. As mentioned in the introduction above. $m$ can be considered as the quantity of tasks in one process. If the shape parameter $m=1$, Erlang distribution simplifies to the exponential distribution.

And other parameters are assumed as follows, Consecutive Delay Cost $C_{P}^{(i)}$ :

$$
\begin{aligned}
& C_{P}^{(1)}=40, C_{P}^{(2)}=80, C_{P}^{(3)}=160, C_{P}^{(4)}=320, \\
& C_{P}^{(5)}=640, C_{P}^{(6)}=1,280, C_{P}^{(7)}=2,560, C_{P}^{(8)}=5,120,
\end{aligned}
$$

$$
\begin{aligned}
& C_{P}^{(9)}=10,240, C_{P}^{(10)}=20,480, C_{P}^{(11)}=40,960, \\
& C_{P}^{(12)}=81,920 \text {, Processes } n=7-12 \text {; Shape Parame- }
\end{aligned}
$$

ter $m=1,2,3,4$; Target Processing Time $Z=2$; Idle Cost $C_{S}=20$.

\subsection{Variation Trend of Expected Cost by Shifting the Allocation of the Untrained Workers}

It showed the variation trend of expected cost by changing the position of untrained one at a time under different disparity of processing speed between worker $A$ and $B$ in the Tables 1 to 3 .

For the first untrained worker, the expected cost reaches lowest when it is arranged at the first period of the processing. And in spite of the disparity of speed of the workers, the monotonic increasing of expected cost unchanged (in Table 1).

And for the second untrained worker, it shows monotonic increasing of expected cost when the disparity of processing speed is small. However, under the condition with great disparity the turning point of expected cost appeared (in Table 2).

For the third untrained worker, same as above, when the disparity is not great, expected cost increases monotonically but reversed when the disparity expands.

\subsection{The Relationship between Optimal Assignment and the Disparity of Workers' Processing Speed}

We can get the optimal assignments by calculating the expecting cost of all assignments under different condition. Table 4 showed the result when shape parameter $m=2$, and processing rate $\mu_{A}=0.1$ and $\mu_{B}$ varies from 0.2 to 2.0 .

Table 4 shows that, despite of the number of processes $n$, the varying patterns are similar. If the disparity of processing rate is small, $\pi(1,2,3)$ is the optimal assignment which means that these three untrained workers are arranged at the first three processes. We call this kind of assignment as centralized optimal assignment.

And when the disparity becomes great, it is optimal that three untrained workers are as far away from each other as possible which can be represented as $\pi\left(1, \frac{1+n}{2}, n\right)$ (when $n$ is odd number) or $\pi\left(1, \frac{n}{2}, n\right)$ (when $n$ is even number). We call this kind of assignment as decentralized optimal assignment.

However when the disparity is between the above two conditions, the expected cost of assignment $\pi(1, x, n)$ is the minimal where $1<x<\frac{1+n}{2}$. And this kind of assignments is called as transition optimal assignment. 
Song, Kong, Yamamoto, Sun, and Matsui: Industrial Engineering \& Management Systems

Vol 14, No 4, December 2015, pp.372-378, (C) 2015 KIIE

Table 1. The changing of expected cost when shift the allocation of the first untrained worker when $m=1$

\begin{tabular}{ccc}
\hline$n=7$ & $\mu_{A}=0.1, \mu_{B}=0.2$ & $\mu_{A}=0.1, \mu_{B}=1.0$ \\
\hline $\boldsymbol{A} B B B B \boldsymbol{A} \boldsymbol{A}$ & 9432.32 & 1686.23 \\
\hline$B \boldsymbol{A} B B B \boldsymbol{A} \boldsymbol{A}$ & 9933.29 & 1742.80 \\
\hline$B B \boldsymbol{B} B B \boldsymbol{A} \boldsymbol{A}$ & 10037.52 & 1797.84 \\
\hline$B B B \boldsymbol{A} B \boldsymbol{A} \boldsymbol{A}$ & 10679.98 & 1959.53 \\
\hline$B B B B \boldsymbol{A} \boldsymbol{A} \boldsymbol{A}$ & 10990.31 & 2545.63 \\
\hline
\end{tabular}

Table 2. The changing of expected cost when shift the allocation of the second untrained worker when $m=1$

\begin{tabular}{ccc}
\hline$n=7$ & $\mu_{A}=0.1, \mu_{B}=0.2$ & $\mu_{A}=0.1, \mu_{B}=1.0$ \\
\hline $\boldsymbol{A} \boldsymbol{A} B B B B \boldsymbol{A}$ & 8255.53 & 1597.15 \\
\hline $\boldsymbol{A} B \boldsymbol{A} B B B \boldsymbol{A}$ & 8586.10 & 1448.07 \\
\hline $\boldsymbol{A} B B \boldsymbol{A} B B \boldsymbol{A}$ & 8875.92 & 1422.57 \\
\hline $\boldsymbol{A} B B B \boldsymbol{A} B \boldsymbol{A}$ & 9150.09 & 1470.54 \\
\hline $\boldsymbol{A}$ BBBBAA & 9432.32 & 1686.23 \\
\hline
\end{tabular}

Table 3. The changing of expected cost when shift the allocation of the third untrained worker when $m=1$

\begin{tabular}{ccc}
\hline$n=7$ & $\mu_{A}=0.1, \mu_{B}=0.2$ & $\mu_{A}=0.1, \mu_{B}=1.0$ \\
\hline $\boldsymbol{A} \boldsymbol{A} \boldsymbol{A} B B B B$ & 7167.89 & 2256.30 \\
\hline $\boldsymbol{A} \boldsymbol{A} B \boldsymbol{A} B B B$ & 7416.34 & 1777.94 \\
\hline $\boldsymbol{A} \boldsymbol{A} B B \boldsymbol{A} B B$ & 7665.71 & 1648.12 \\
\hline $\boldsymbol{A} \boldsymbol{A} B B B \boldsymbol{A} B$ & 7937.60 & 1611.71 \\
\hline $\boldsymbol{A} \boldsymbol{A} B B B B \boldsymbol{A}$ & 8255.53 & 1597.15 \\
\hline
\end{tabular}

4.3 The Relationship between Optimal Assignment and the Shaping Parameter

Table 5 shows the influence of shaping parameter to the optimal assignment. By numerical experiment under the same processing process $n=9$, and setting the processing rate of untrained worker $\mu_{A}$ to 0.1 , four groups of data with variable shaping parameter $m$ are listed by ascending order of $\mu_{B}$.

From Table 5, we can realize that no matter how

Table 4. Optimal assignment when $m=2, \mu_{A}=0.1, \mu_{B}$ varies from 0.2 to 2.0 under 7 to 12 processes

\begin{tabular}{cc|cccccc}
\hline$\mu_{A}$ & $\mu_{B}$ & $n=7$ & $n=8$ & $n=9$ & $n=10$ & $n=11$ & $n=12$ \\
\hline 0.1 & 0.2 & AAABBBB & AAABBBBB & AAABBBBBB & AAABBBBBBB & AAABBBBBBBB & AAABBBBBBBBB \\
\hline 0.1 & $0.3-0.8$ & AAABBBB & AAABBBBB & AAABBBBBB & AAABBBBBBB & AAABBBBBBBB & AAABBBBBBBBB \\
\hline 0.1 & 0.9 & AAABBBB & AAABBBBB & AAABBBBBB & AAABBBBBBB & AAABBBBBBBB & AAABBBBBBBBB \\
\hline 0.1 & 1.0 & AABBBBA & AABBBBBA & AABBBBBBA & AABBBBBBBA & AABBBBBBBBA & AABBBBBBBBBA \\
\hline 0.1 & 1.1 & AABBBBA & AABBBBBA & AABBBBBBA & AABBBBBBBA & AABBBBBBBBA & AABBBBBBBBBA \\
\hline 0.1 & 1.2 & AABBBBA & AABBBBBA & ABABBBBBA & ABABBBBBBA & ABBABBBBBBA & ABBABBBBBBBA \\
\hline 0.1 & 1.3 & ABABBBA & ABABBBBA & ABBABBBBA & ABBABBBBBA & ABBBABBBBBA & ABBBABBBBBBA \\
\hline 0.1 & 1.4 & ABABBBA & ABBABBBA & ABBABBBBA & ABBBABBBBA & ABBBABBBBBA & ABBBBABBBBBA \\
\hline 0.1 & 1.5 & ABBABBA & ABBABBBA & ABBBABBBA & ABBBABBBBA & ABBBBABBBBA & ABBBBABBBBBA \\
\hline 0.1 & 1.6 & ABBABBA & ABBABBBA & ABBBABBBA & ABBBABBBBA & ABBBBABBBBA & ABBBBABBBBBA \\
\hline 0.1 & 1.7 & ABBABBA & ABBABBBA & ABBBABBBA & ABBBABBBBA & ABBBBABBBBA & ABBBBABBBBBA \\
\hline 0.1 & $1.8-2.0$ & ABBABBA & ABBABBBA & ABBBABBBA & ABBBABBBBA & ABBBBABBBBA & ABBBBABBBBBA \\
\hline
\end{tabular}

Table 5. Optimal assignment when $n=9, \mu_{A}=0.1, \mu_{B}$ varies from 0.2 to 2.0 under shaping parameter varying from 1 to 4

\begin{tabular}{cccccccccccc|c}
\hline$\mu_{A}$ & $\mu_{B}$ & $m=1$ & $\mu_{A}$ & $\mu_{B}$ & $m=2$ & $\mu_{A}$ & $\mu_{B}$ & $m=3$ & $\mu_{A}$ & $\mu_{B}$ & $m=4$ \\
\hline 0.1 & 0.2 & AAABBBBBB & 0.1 & 0.8 & AAABBBBBB & 0.1 & 1.3 & AAABBBBBB & 0.1 & 1.9 & AAABBBBBB \\
\hline 0.1 & 0.3 & AAABBBBBB & 0.1 & 0.9 & AAABBBBBB & 0.1 & 1.4 & AAABBBBBB & 0.1 & $\mathbf{2 . 0}$ & AABBBBBBA \\
\hline 0.1 & $\mathbf{0 . 4}$ & AABBBBBBA & 0.1 & $\mathbf{1 . 0}$ & AABBBBBBA & 0.1 & $\mathbf{1 . 5}$ & AABBBBBBA & 0.1 & $\mathbf{2 . 1}$ & AABBBBBBA \\
\hline 0.1 & $\mathbf{0 . 5}$ & AABBBBBBA & 0.1 & $\mathbf{1 . 1}$ & AABBBBBBA & 0.1 & $\mathbf{1 . 6}$ & AABBBBBBA & 0.1 & $\mathbf{2 . 2}$ & AABBBBBBA \\
\hline 0.1 & $\mathbf{0 . 6}$ & AABBBBBBA & 0.1 & $\mathbf{1 . 2}$ & ABABBBBBA & 0.1 & $\mathbf{1 . 7}$ & AABBBBBBA & 0.1 & $\mathbf{2 . 3}$ & AABBBBBBA \\
\hline 0.1 & $\mathbf{0 . 7}$ & ABBABBBBA & 0.1 & $\mathbf{1 . 3}$ & ABBABBBBA & 0.1 & $\mathbf{1 . 8}$ & ABABBBBBA & 0.1 & $\mathbf{2 . 4}$ & ABABBBBBA \\
\hline 0.1 & 0.8 & ABBBABBBA & 0.1 & $\mathbf{1 . 4}$ & ABBABBBBA & 0.1 & $\mathbf{1 . 9}$ & ABBABBBBA & 0.1 & $\mathbf{2 . 5}$ & ABBABBBBA \\
\hline 0.1 & 0.9 & ABBBABBBA & 0.1 & 1.5 & ABBBABBBA & 0.1 & $\mathbf{2 . 0}$ & ABBABBBBA & 0.1 & $\mathbf{2 . 6}$ & ABBABBBBA \\
\hline 0.1 & 1.0 & ABBBABBBA & 0.1 & 1.6 & ABBBABBBA & 0.1 & 2.1 & ABBBABBBA & 0.1 & 2.7 & ABBBABBBA
\end{tabular}


the shaping parameter changes, the three kinds of optimal assignment is invariant. By another word, we can assume that the new kind of optimal assignment will not appear and these three kinds of optimal assignment will also not disappear in any value of shaping parameter $m$. And this inspires us that these three kinds of optimal assignment is constant even when workers' processing speed follows other distribution, such as Gamma distribution. And in the future researches, we will try to demonstrate this as a law by mathematical proof.

However, when $m=1$, the centralized optimal distribution appears until $\mu_{B}$ is 0.3 , and when $m$ increase to 4 , the centralized optimal distribution was expanded to until $\mu_{B}$ is 1.9. And also the occurrence of transition and decentralized optimal assignment is later.

\subsection{The variation trend of the boundary of optimal Assignment by the Experiment of Changing the Both Values of $\mu_{A}, \mu_{B}$}

Table 6 and Table 7 are the result of numerical experiments under $m=2$ and $n=7$ changing both of values of processing rate of well-trained workers and un- trained workers.

The variation trend of the boundary of optimal assignment is obviously revealed. From Table 6, we can see when $\mu_{A}=0.1$, the transition optimal assignment will not appear until $\mu_{B}$ increasing to 1.0 . However, by setting $\mu_{A}$ to 0.7 , the transition optimal assignment appears even when $\mu_{B}$ is 0.8 , which means there is not a striking difference of processing speed between these two kinds of workers. We can summarize with the increasing of $\mu_{A}$, the processing rate of untrained workers, the transition optimal assignment appears earlier when $\mu_{A}$ is almost the same as $\mu_{B}$.

On the contrary, the boundary of transition and decentralized optimal assignment shows disparate variation trend. With the increasing of value of $\mu_{A}$, the appearance of decentralized optimal assignment needs a larger value of $\mu_{B}$. For example, when $\mu_{A}$ is 1.2 , the appearance of decentralized optimal assignment needs $\mu_{B}$ is larger than 1.6 , but when $\mu_{A}$ is 1.3 , the appearance of decentralized optimal assignment needs $\mu_{B}$ is larger than 1.7.

Although these two boundaries show different trend, each of them monotonically increases or decrease. It suggest us the monotony may be influenced by the mono-

Table 6. Boundary of centralized and transition optimal assignment when $n=7, m=2$

\begin{tabular}{|c|c|c|c|c|c|c|c|c|c|}
\hline$\mu_{\mathrm{B}} \mu_{\mathrm{A}}$ & 0.1 & 0.2 & 0.3 & 0.4 & 0.5 & 0.6 & 0.7 & 0.8 & $\cdots$ \\
\hline$\cdots$ & $\cdots$ & $\cdots$ & $\cdots$ & $\cdots$ & $\cdots$ & $\cdots$ & $\cdots$ & $\cdots$ & $\cdots$ \\
\hline 0.5 & AAABBBB & AAABBBB & AAABBBB & AAABBBB & $x$ & $x$ & $x$ & $x$ & $\cdots$ \\
\hline 0.6 & AAABBBB & AAABBBB & AAABBBB & AAABBBB & AAABBBB & $\times$ & $x$ & $x$ & $\cdots$ \\
\hline 0.7 & AAABBBB & AAABBBB & AAABBBB & AAABBBB & AAABBBB & AAABBBB & $x$ & $x$ & $\cdots$ \\
\hline 0.8 & AAABBBB & AAABBBB & AAABBBB & AАABBBB & AABBBBA & AABBBBA & AABBBBA & $x$ & $\cdots$ \\
\hline 0.9 & AAABBBB & AAABBBB & AAABBBB & AABBBBA & AABBBBA & AABBBBA & AABBBBA & AABBBBA & $\cdots$ \\
\hline 1.0 & AABBBBA & AABBBBA & AABBBBA & AABBBBA & AABBBBA & AABBBBA & AABBBBA & AABBBBA & $\cdots$ \\
\hline 1.1 & AABBBBA & AABBBBA & AABBBBA & AABBBBA & AABBBBA & AABBBBA & AABBBBA & AABBBBA & $\cdots$ \\
\hline$\cdots$ & $\cdots$ & $\cdots$ & $\cdots$ & $\cdots$ & $\cdots$ & $\cdots$ & $\cdots$ & $\cdots$ & $\cdots$ \\
\hline
\end{tabular}

Table 7. Boundary of transition and decentralized optimal assignment when $n=7, m=2$

\begin{tabular}{|c|c|c|c|c|c|c|c|c|c|c|}
\hline$\mu_{\mathrm{B}} \mu_{\mathrm{A}}$ & 0.1 & $\ldots$ & 1.0 & 1.1 & 1.2 & 1.3 & 1.4 & 1.5 & 1.6 & $\ldots$ \\
\hline$\cdots$ & $\cdots$ & $\cdots$ & $\cdots$ & $\cdots$ & $\cdots$ & $\cdots$ & $\cdots$ & $\cdots$ & $\cdots$ & $\ldots$ \\
\hline 1.4 & ABABBBA & $\cdots$ & ABABBBA & AABBBBA & AABBBBA & AABBBBA & $x$ & $x$ & $x$ & $\cdots$ \\
\hline 1.5 & ABBABBA & $\cdots$ & ABBABBA & ABABBBA & AABBBBA & AABBBBA & AABBBBA & $\times$ & $x$ & $\cdots$ \\
\hline 1.6 & ABBABBA & $\cdots$ & ABBABBA & ABBABBA & ABBABBA & ABABBBA & ABABBBA & AABBBBA & $x$ & $\cdots$ \\
\hline 1.7 & ABBABBA & $\cdots$ & ABBABBA & ABBABBA & ABBABBA & ABBABBA & ABBABBA & AАВBBBA & AABBBBA & $\cdots$ \\
\hline 1.8 & ABBABBA & $\cdots$ & ABBABBA & ABBABBA & ABBABBA & ABBABBA & ABBABBA & ABBABBA & ABABBBA & $\cdots$ \\
\hline 1.9 & ABBABBA & $\cdots$ & ABBABBA & ABBABBA & ABBABBA & ABBABBA & ABBABBA & ABBABBA & ABBABBA & $\cdots$ \\
\hline 2.0 & ABBABBA & $\cdots$ & ABBABBA & ABBABBA & ABBABBA & ABBABBA & ABBABBA & ABBABBA & ABBABBA & $\cdots$ \\
\hline$\cdots$ & $\cdots$ & $\cdots$ & $\cdots$ & $\cdots$ & $\cdots$ & $\cdots$ & $\cdots$ & $\cdots$ & $\cdots$ & $\ldots$ \\
\hline
\end{tabular}


tonically variation trend of other factors, such as the expected cost. As a future research, we will verify the affecting factors of the optimal assignment by mathematical demonstration.

\section{CONCLUSION}

In this paper, we considered the properties of optimal assignment with three untrained workers in LCMw MP. First, we systematically classified and modeled the multi-period problem and defined the optimal workers assignment problem under the reset model. Secondly, by the conclusion of optimal worker assignment with two untrained workers (Kong et al., 2011a, 2011b), we assume the assignment of the case of three untrained workers and finally, by analyzing the results of mathematical experiments, we get the following conclusions.

1. In spite of the value of shaping parameter $m$ and number of process $n$, the three types of optimal assignment is constant.

We call $\pi(1,2,3)$ centralized optimal assignment which means the three untrained workers are arranged at the first three processes. And $\pi(1, x, n)$, where $1<x<\frac{1+n}{2}$, is called as transition optimal assignment, which implies that two of the untrained workers are assigned at the head and tail of the processing line and another one is assigned at the first half of the line. Finally, $\pi\left(1, \frac{1+n}{2}, n\right)$ (when $n$ is odd number) or $\pi\left(1, \frac{n}{2}, n\right)$ (when $n$ is even number) is the type of decentralized optimal assignment, which suggests that it is optimal when three untrained workers are arranged as far as possible from each other.

2. When both of processing rates between two kinds of workers are not high enough, centralized assignment $\pi(1,2,3)$ is the optimal worker assignment.

When the processing speed of regular worker is fast enough to remedy the delay caused by the untrained workers, decentralized assignment $\pi\left(1, \frac{1+n}{2}, n\right)$ (when $n$ is odd number) or $\pi\left(1, \frac{n}{2}, n\right)$ (when $n$ is even number) will be the optimal assignment. And besides these, assignment $\pi(1, x, n)$, where $1<x<\frac{1+n}{2}$ is the transition of these two situations.

As a goal of future researches, we will try to verify the affecting factors of the optimal assignment by mathematical demonstration. It is also clear that the optimal assignment is strongly influenced by the relation between increasing rate of consecutive delay cost and the disparity of two kinds of workers and we will search for the property of it by theoretically demonstration.

\section{REFERENCES}

Kong, X., Sun, J., Yamamoto, H., and Matsui, M. (2010), A Study of an optimal arrangement of a processing system with two kinds of workers in a limited-cycle problem with multiple periods, presented at the 11th Asia Pacific Industrial Engineering and Management Systems Conference (APIEMS), Melaka, Malaysia.

Kong, X., Sun, J., Yamamoto, H., and Matsui, M. (2011a), Two special Workers' optimal assignment with two kinds of workers under a limited-cycle problem with multiple periods, presented at the 21st International Conference of Production Research (ICPR), Stuttgart, Germany.

Kong, X., Sun J., Yamamoto H., and Matsui M. (2011b), Optimal worker assignment with two special workers in limited-cycle multiple periods, presented at the 2011 Asian Conference of Management Science and Applications (ACMSA), Sanya, China.

Nils, B., Malte, F., and Armin, S. (2007), A classification of assembly line balancing problems, European Journal of Operational Research, 183, 674693.

Salveson, M. E. (1955), The assembly line balancing problem, The Journal of Industrial Engineering, 6(3), 18-25.

Song, P., Kong, X., Yamamoto, H., Sun, J., Matsui, M. (2014), A Study of Optimal Worker Assignment with Three Rookies under a Limited-Cycle Model with Multiple Periods, Presented at the 12th International Conference on Industrial Management (ICIM), Chengdu, China.

Yamamoto, H., Matsui, M., and Liu, J. (2006), A basic study on a limited-cycle problem with multi periods and the optimal assignment problem, Journal of Japan Industrial Management Association, 57, 23-31.

Yamamoto, H., Matsui, M., and Bai, X. S. (2007), A branch and bound method for the optimal assignment during a Limit-cycle problem with multiple periods, Journal of Japan Industrial Management Association, 58, 37-43.

Yamamoto, H., Sun, J., and Matsui, M. (2010), A study on limited-cycle scheduling problem with multiple periods, Computer and Industrial Engineering, 59 (4), 675-681.

Yamamoto, H., Sun, J., Matsui, M., and Kong, X. D. (2011), A study of the optimal arrangement in the reset limited-cycle problem with multiple periods: with fewer special workers, Journal of Japan Industrial Management Association, 62(5), 239-246. 